\title{
AZGP1 suppresses the process of colorectal cancer after upregulating FASN expression via mTOR signal pathway
}

\author{
Wenxiu Yu*, Jun Ling*, Hailin Yu, Jiang Du and Ting Liu \\ Department of Gastroenterology, The Affiliated Jurong Hospital to Jiangsu University, Jurong, Jiangsu, P. R. China
}

\begin{abstract}
Colorectal cancer (CRC) is the most common malignant gastrointestinal tumor. Obesity has been confirmed to be closely related to the occurrence of CRC, but the specific mechanism is not clear. This study mainly explored the roles of obesity-related genes, fatty acid synthase (FASN) and zinc-alpha-2-glycoprotein (AZGP1) in CRC. 30 cases of CRC tissues and adjacent normal colorectal tissues were obtained to quantify the levels of FASN and AZGP1 using qRT-PCR and Western blotting. Overexpression-AZGP1, overexpression-FASN and FASN shRNA were transfected into SW480 cells. CCK-8, wound healing and transwell assays were used to evaluate the roles of FASN and AZGP1 on cell proliferation, migration as well as invasion. Western blot was performed to investigate the expression of MMP-2, MMP-9 and mTOR signaling-related proteins. AZGP1 expression was decreased in CRC tissues, which was negatively correlated with FASN expression. Overexpression-AZGP1 showed a significant inhibitory effect on cell proliferation, invasion and migration via inhibiting MMP-2 and MMP-9 expressions. Furthermore, up-regulation of AZGP1 suppressed the expression of mTOR pathway downstream proteins 4EBP and eIF4E through inhibiting FASN expression. Reintroduction of overexpression-FASN could partially reverse and inhibition of FASN further decrease the antitumor effect of AZGP1. AZGP1 suppresses CRC cellular activities by regulating FASN via mTOR pathway, suggesting that AZGP1 and FASN may be the targets for CRC therapy.
\end{abstract}

Key words: Colorectal cancer - AZGP1 - Fatty acid synthase - mTOR

\section{Introduction}

At present, the malignant phenotype of most tumor tissues is closely related to the metabolisms of special material and energy, such as oral cancer (Nakazato et al. 2019), hepatocellular carcinoma (Fang et al. 2019), pancreatic cancer (Yang et al. 2019), non-small cell lung cancer (Zhang et al. 2019), prostate cancer (Marin de Mas et al. 2019), etc. Colorectal cancer (CRC) is the most frequent malignant gastrointestinal tumor and a leading cause of cancer death worldwide, along with an increase of about 150,000 cases every year (Jemal et al. 2008). The links between obesity and metabolisms are well known. Obesity, a clear premature lesion of CRC, is also closely related to CRC occurrence and development (Lars-

\footnotetext{
* These authors contributed equally.

Correspondence to: Hailin Yu, Department of Gastroenterology, The Affiliated Jurong Hospital to Jiangsu University, No.60 Xi Road, Jurong, Jiangsu, 212400, P. R. China

E-mail: YuHailin1201@163.com
}

son and Wolk 2007; Aleksandrova et al. 2017). However, the underlying mechanisms between them are still unclear.

Fatty acid synthase (FASN) is a key enzyme in the synthesis of endogenous fatty acids, which combines with acetyl-CoA, malonyl-CoA and NADPH as the substrate to synthesize palmitate (Cui et al. 2017). Recent studies have shown that FASN is over-expressed in most tumors and its activity is required for the malignant biological behaviors of tumor cells, and FASN mediating metabolic abnormalities participates in the occurrence and development of a variety of tumors, such as breast cancer (Wang et al. 2001), colon cancer (Zaytseva et al. 2012), ovarian cancer (Cai et al. 2015), etc. Previously, it was found that FASN gradually increased from cell proliferation, atypical hyperplasia to malignant hyperplasia in CRC (Ogino et al. 2007), suggesting that high expression of FASN was closely associated to the occurrence and development of CRC, and high FASN expression might be one of the specific molecular markers of CRC. FASN expression is provided to be regulated by zinc- $\alpha 2$-glycoprotein (AZGP1) (Chang et al. 2019), which is modulated by mTOR 
signaling pathway (Chang et al. 2014). The mTOR signaling pathway is primarily regulated by upstream PI3K/Akt and AMPK signaling pathways via activating the phosphorylation of 4E-BP1 and S6, thereby cell growth and metabolism are accelerated (Gao et al. 2004; Nozawa et al. 2007). It has been reported that the mTOR signaling pathway is activated in about $40 \%$ of patients with CRC (Nozawa et al. 2007). Notably, the expression of FASN is down-regulated after inhibition of Akt/mTOR pathway (Li et al. 2012). FASN is involved in glucose metabolism via down-regulation of the AKT/ERK pathway and eventually alters the malignant phenotypes in lung cancer cells (Chang et al. 2019). Therefore, the exact interaction between the mTOR signaling pathway and FASN is worth exploring.

AZGP1 is mostly expressed in the epithelial cells of breast, prostate, liver and various gastrointestinal organs (Tada et al. 1991). Low serum AZGP1 levels are strongly associated with obesity (Marrades et al. 2008). Recent studies have shown that AZGP1 is also closely correlated with tumorigenesis and poor tumor differentiation in prostate cancer and breast cancer (Diez-Itza et al. 1993; Hale et al. 2001). The amino acid sequence of AZGP1 is similar to that of the tumor-derived fat mobilization factor, which can promote the decomposition of fat cells, leading to cachexia in patients with tumor (Russell et al. 2004). We speculate that AZGP1 may participate in tumorigenesis and development by regulating energy metabolism pathways.

AZGP1 and FASN appear to play important roles not only in glucose and lipid metabolism but also in the development and progression of several obesity-related malignancies. The main aim of the present study is to investigate whether obesity-related genes FASN and AZGP1 regulate malignant phenotypes of CRC cells through mTOR signaling pathway, and finally provide valuable targets and theoretical basis for selective targeted therapy for patients with clinical CRC.

\section{Materials and Methods}

\section{Clinical tissues collection}

Total 30 cases of CRC tissues and adjacent normal colorectal tissues were obtained from the Affiliated Jurong Hospital to Jiangsu University. The tissues were immediately frozen in liquid nitrogen for subsequent RNA or protein extraction. All the enrolled patients were notified of this research purpose and written informed consents. This study was approved by the medical Ethics Committee of the Affiliated Jurong Hospital to Jiangsu University.

\section{Cells culture}

Four human CRC cell lines (SW620, SW480, HT-29 and HCT116), and human normal colonic epithelial cell line
(NCM460) were purchased from the Chinese Academy of Sciences Type Culture Collection (Shanghai, China). Cells were cultured in RPMI 1640 medium (Gibco, Carlsbad, CA) added with $10 \%$ fetal bovine serum (Gibco, GrandIsland, NY) and 1\% penicillin/streptomycin solution (Termo Fisher Scientifc, Rockford, IL). Cells were incubated in a $5 \% \mathrm{CO}_{2}$ incubator at $37^{\circ} \mathrm{C}$.

\section{Establishing stable cell lines, cell transfection and grouping}

The plasmids of overexpression-AZGP1, overexpressionFASN, overexpression negative control (overexpressionNC), FASN short hairpin RNA (shRNA) and shRNA NC were constructed by RiboBio (GuangZhou, China). According to the manufacturer's instructions, overexpression-AZGP1, overexpression-FASN and overexpressionNC were transfected into SW480 cells for 48 h using Lipofectamine 2000 reagent (Invitrogen, Carlsbad, CA). All plasmids were used at a final concentration of $45 \mathrm{nM}$. Here, SW480 cells overexpressed with AZGP1 and FASN are stable cell line.

First, experimental groups were as follows: control, overexpression-NC and overexpression-AZGP1 groups. Then, cells transfected with overexpression-AZGP1 were incubated with rapamycin (30 $\mathrm{nM}$, inhibitor of mTOR) for $48 \mathrm{~h}$ and experimental groups were as follows: control (Con), overexpression-NC (NC), overexpression-AZGP1 (AZGP1), overexpression-NC + rapamycin (NC+Rap) and overexpression-AZGP1 + rapamycin (AZGP1+Rap) groups. Subsequently, cells were transfected with overexpressionFASN or co-transfected with overexpression-FASN + overexpression-AZGP1 or FASN shRNA + overexpression-AZGP1, and experimental groups were as follows: control (Con), overexpression-NC (NC), overexpression-AZGP1 (AZGP1), overexpression-FASN (FASN), overexpression-AZGP1 + FASN (AZGP1+FASN) and overexpression-AZGP1 + FASN shRNA (AZGP1+sh-FASN) groups.

\section{Cell proliferation assay}

For the Cell Counting Kit-8 (CCK-8) assay, SW480 cells were seeded in 96-well plates. After cell transfection, cells were cultured for 24, 48 and $72 \mathrm{~h}$, respectively, before conducting the CCK-8 assay (Dojindo, Japan). After $1 \mathrm{~h}$ of incubation with CCK-8 solution at $37^{\circ} \mathrm{C}$, the absorbance at a wavelength of $450 \mathrm{~nm}$ was detected and used for determining cell proliferation.

\section{Wound healing assay}

After transfection, cells $\left(1 \times 10^{5}\right.$ cells/well $)$ were collected and seeded into 6-well plates, and then cultured to reach $100 \%$ confluence. Across the center of the well, the cell mon- 
olayer was scratched with a $10 \mu$ pipette tip. Subsequently, these cells were cultured for $72 \mathrm{~h}$. An inverted microscope (Olympus, Japan) was used for observation and photography, and images of the wound areas were analyzed using ImageJ software.

\section{Transwell assay}

After transfection, cells $\left(1 \times 10^{5}\right.$ cells/well) were suspended in 6-well plates (Thermo Fisher Scientific, Inc.). $300 \mu \mathrm{l}$ of cell suspension was added into each of the upper wells of Transwell invasion chamber. Cells were then incubated for $72 \mathrm{~h}$ at $37^{\circ} \mathrm{C}$ with $5 \% \mathrm{CO}_{2}$. Subsequently, cells on the lower membrane of the inserts were fixed in $4 \%$ paraformaldehyde at $4^{\circ} \mathrm{C}$ and stained with $0.05 \%$ crystal violet for $10 \mathrm{~min}$ at room temperature. Images of at least four randomly selected fields in per membrane were captured under a microscope and the numbers of invaded cells were quantified using ImageJ software (National Institutes of Health, USA).

\section{RNA isolation and $q R T-P C R$}

Total RNA was isolated using TRIzol reagent (Invitrogen). qRT-PCR was performed using the Applied Biosystems ABI Prism 7000 Sequence Detection System using the SYBR Green PCR master mix (ABI catalog \#4309155). Primers used in qRT-PCR were exhibited as follows: AZGP1, forward 5'-TACAACGACAGTAACGGGTCT-3', reverse
5'-TATTTCCAGAATGCTCCGCTG-3'; FASN, forward 5'-AAGGACCTGTCTAGGTTTGATGC-3', reverse 5'-TGGCTTCATAGGTGACTTCCA-3'. GAPDH was used to normalize the mRNA expression of these genes. PCR results were normalized and data were calculated by the $2^{-\Delta \Delta \mathrm{ct}}$ method.

\section{Western blot analysis}

Cell protein samples were loaded onto a 10\% SDS-PAGE and then transferred to PVDF membranes (Bio-Rad, Hercules, CA). Antibodies against AZGP1, FASN, MMP-2, MMP-9, p-mTOR, mTOR, p-4EBP, 4EBP, eIF4E and GAPDH (1:1000) were all obtained from Abcam. Secondary HRP-conjugated antibodies (1:2000, Santa Cruz, Delaware, CA) were used and the protein bands were visualized using ECL chemiluminescent substrate (GE, Buckinghamshire, UK).

\section{Statistical analysis}

Data were presented as mean \pm SD and experiments were carried out for at least three times. SPSS 19.0 software (SPSS Inc., Chicago, USA) was performed to do statistical analysis. Comparisons between quantitative variables were analyzed by Student's $t$-test and One-way ANOVA analysis, and correlations between variables were examined using Pearson's correlation coefficient. A $p$-value $<0.05$ is considered statistically significant.
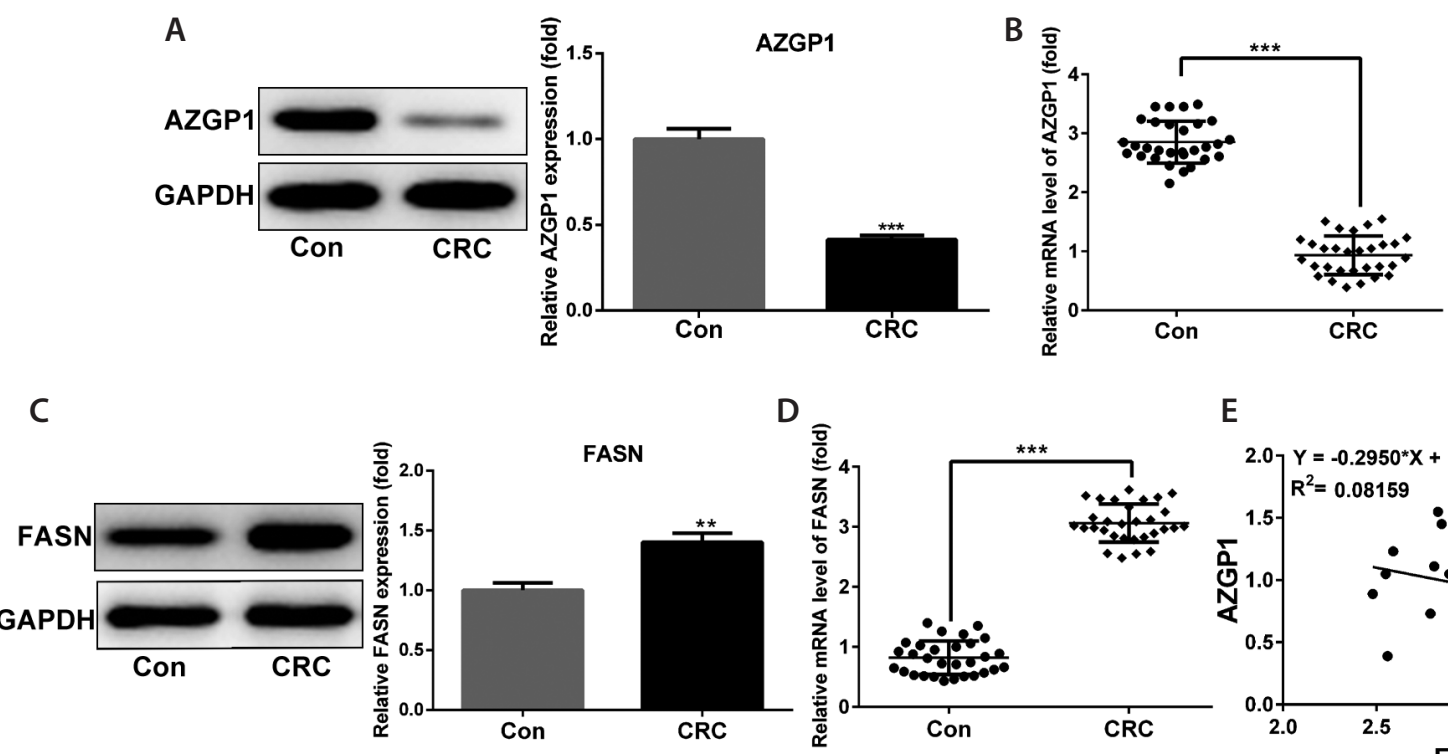

E

Figure 1. The expression levels of AZGP1 and FASN in colorectal cancer (CRC) tissues. A. AZGP1 protein expression in CRC tissues was measured by Western blot. B. The relative mRNA expression levels of AZGP1 in 30 cases of CRC tissues were examined by qRT-PCR. FASN protein expression (C) and mRNA expression (D) in 30 cases of CRC tissues were analyzed through Western blot and qRT-PCR. E. Pearson's correlation analysis of the relationship between AZGP1 and FASN. ${ }^{* *} p<0.01,{ }^{* *} p<0.01$ vs. control (Con). 


\section{Results}

AZGP1 and FASN expression in tissue samples and adjacent control samples from CRC patients, and the correlation between the two genes

First, the protein and mRNA levels of AZGP1 and FASN in tissue samples and adjacent control tissues $(n=30)$ obtained from CRC patients were measured. It was identified that the protein and mRNA levels of AZGP1 were remarkably reduced in cancerous tissues ( $p<0.001$, Fig. $1 \mathrm{~A}$ and B), while FASN mRNA and protein expression was significantly elevated in the CRC tissues compared with the control tissues $(p<0.01$ and $p<0.001$; Fig. $1 \mathrm{C}$ and D). Pearson's correlation analysis revealed a slightly negative correlation between AZGP1 and FASN $\left(\mathrm{R}^{2}=0.08159\right.$; Fig. 1E). Taken together, AZGP1 and FASN were decreased and increased in CRC tissues, respectively, and a negative association between them was discovered.

\section{AZGP1 expression in CRC cell lines and a normal cell line}

Based on above results, the following experiments focused on the expression of AZGP1 in cell lines in vitro so as to explore its role and underlying mechanisms. The protein and mRNA levels of AZGP1 were therefore detected in different CRC cell lines. The Western blot and qRT-PCR results indicated an apparent decrease of AZGP1 expression in the CRC cell lines (SW620, SW480, HT-29 and HCT116) compared with that in the NCM460 cell line $(p<0.05, p<0.01$ and $p<0.001$; Fig. $2 \mathrm{~A}$ and B). The SW480 cell line exhibited the most marked downexpression of AZGP1, and thus, SW480 cells were selected for subsequent experiments. Next, the results of Western blot and qRT-PCR discovered a marked increase both in protein and mRNA levels of AZGP1 in the overexpression-AZGP1 group when compared with those in the control and overexpressionNC groups ( $p<0.001$; Fig. $2 \mathrm{C}$ and D). These findings illustrated that AZGP1 was under-expressed in CRC cells.

\section{Effects of overexpression-AZGP1 on multiple malignant phenotypes of SW480 cells}

The CCK-8 assay revealed that overexpression-AZGP1transfected SW480 cells exhibited a significantly decreased growth rate compared with that of the overexpressionNC-transfected cells and control cells $(p<0.01$ at $48 \mathrm{~h}, p<$ 0.001 at 72 h; Fig. 3A). Here, overexpression of AZGP1 was indicated to markedly reduce the proliferation/survival rate of SW480 cells. The migration and invasion abilities
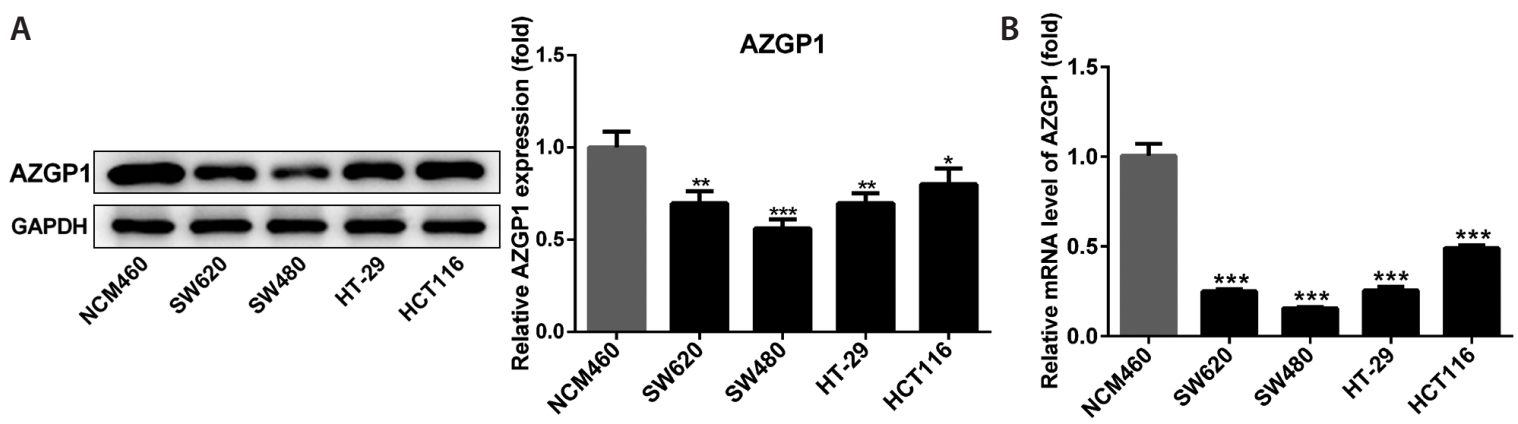

C
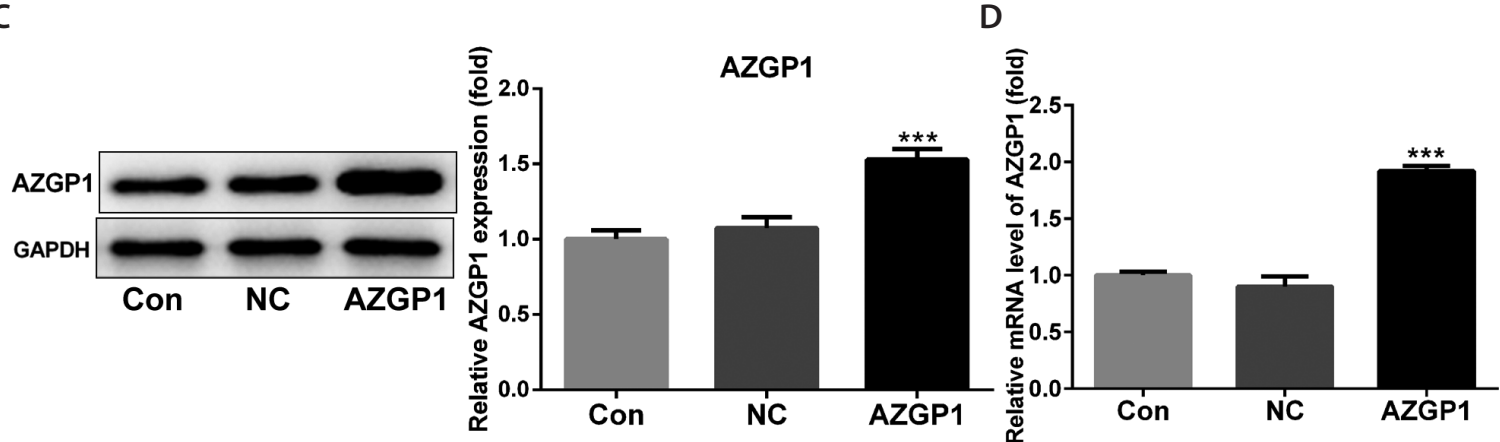

Figure 2. The expression levels of AZGP1 in colorectal cancer cell lines. A, B. Western blot and RT-qPCR found the low protein and mRNA levels of AZGP1 in four human colon cancer cell lines, including SW620, SW480, HT-29 and HCT116, compared with human normal colonic epithelial cells, NCM460. ${ }^{\star} p<0.05,{ }^{* *} p<0.01,{ }^{* *} p<0.001 v s$. NCM460. C, D. Western blot and RT-qPCR indicated the protein and mRNA levels of AZGP1 in SW480 cells after transfection with overexpression-AZGP1 (AZGP1) and overexpression negative control (NC). ${ }^{* * *} p<0.001$ vs. control (Con). 


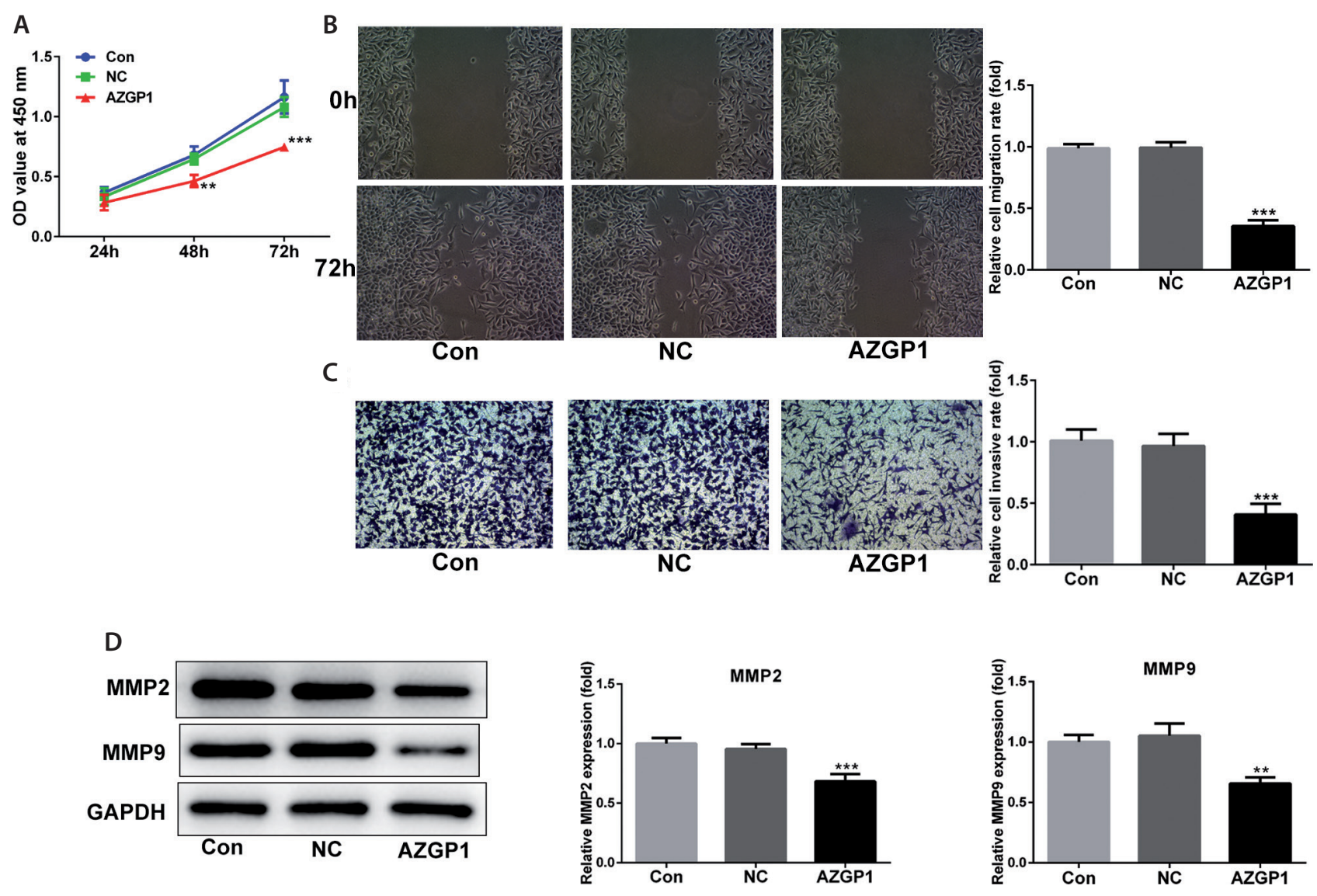

Figure 3. AZGP1 overexpression inhibited cell proliferation, invasion and migration by MMP-2 and MMP-9 proteins. A. The effect of AZGP1 overexpression on cell proliferation using Cell Counting Kit-8 (CCK-8) assay. B. The impact of AZGP1 upregulation on cell migration by wound healing assays. C. The effect of AZGP1 overexpression on cell invasion by Transwell assays. D. The protein expressions of MMP-2 and MMP-9 were determined using Western blot. ${ }^{*} p<0.01,{ }^{* * *} p<0.001 v s$. control (Con). NC, negative control.

of SW480 cells transfected with overexpression-AZGP1 decreased obviously when compared with that of the cells in the control and overexpression-NC groups $(p<0.001$; Fig. 3B and $C$ ). These results demonstrated that AZGP1 had a key role in the progression of CRC through affecting cellular activities.

\section{Expression of MMP-2, MMP-9, p/t-mTOR, eIF4E and $p / t-4 E B P 1$ in $S W 480$ cells}

In order to investigate whether AZGP1 was involved in CRC through mTOR signal pathway, the effect of AZGP1 on the expression of MMP-2, MMP-9, p/t-mTOR, eIF4E and p/t4EBP1 was then assessed. As presented in Fig. 3D, Western blot analysis revealed that the expression of MMP-2 and MMP-9 was significantly decreased following AZGP1 overexpression. Similarly, significant decreases in the protein levels of p-mTOR, eIF4E and p-4EBP1 were observed following AZGP1 overexpression ( $p<0.01$ and $p<0.001$; Fig. 4A). AZGP1 was proved to suppress the downstream proteins of mTOR pathway, including eIF4E and p-4EBP1. These results showed that AZGP1 negatively regulate the mTOR signaling pathway.

\section{Effect of overexpression-AZGP1 and $m$ TOR inhibitor (rapamycin) on FASN expression in SW480 cells}

The results of Western blot showed that the protein levels of FASN were notably reduced following AZGP1 overexpression or rapamycin addition when compared with the control or overexpression-NC group ( $p<0.05$; Fig. $4 \mathrm{~B}$ ), and a dramatic decrease in the protein level of FASN was observed following mTOR knockdown with rapamycin plus overexpression-AZGP1 ( $p<0.01$; Fig. 4B). Inversely, rapamycin could elevate the AZGP1 expression remarkably $(p<0.01$ and $p<0.001$; Fig. 4C). These findings confirmed that FASN and AZGP1 might be engaged in the regulation of mTOR signaling pathways. 

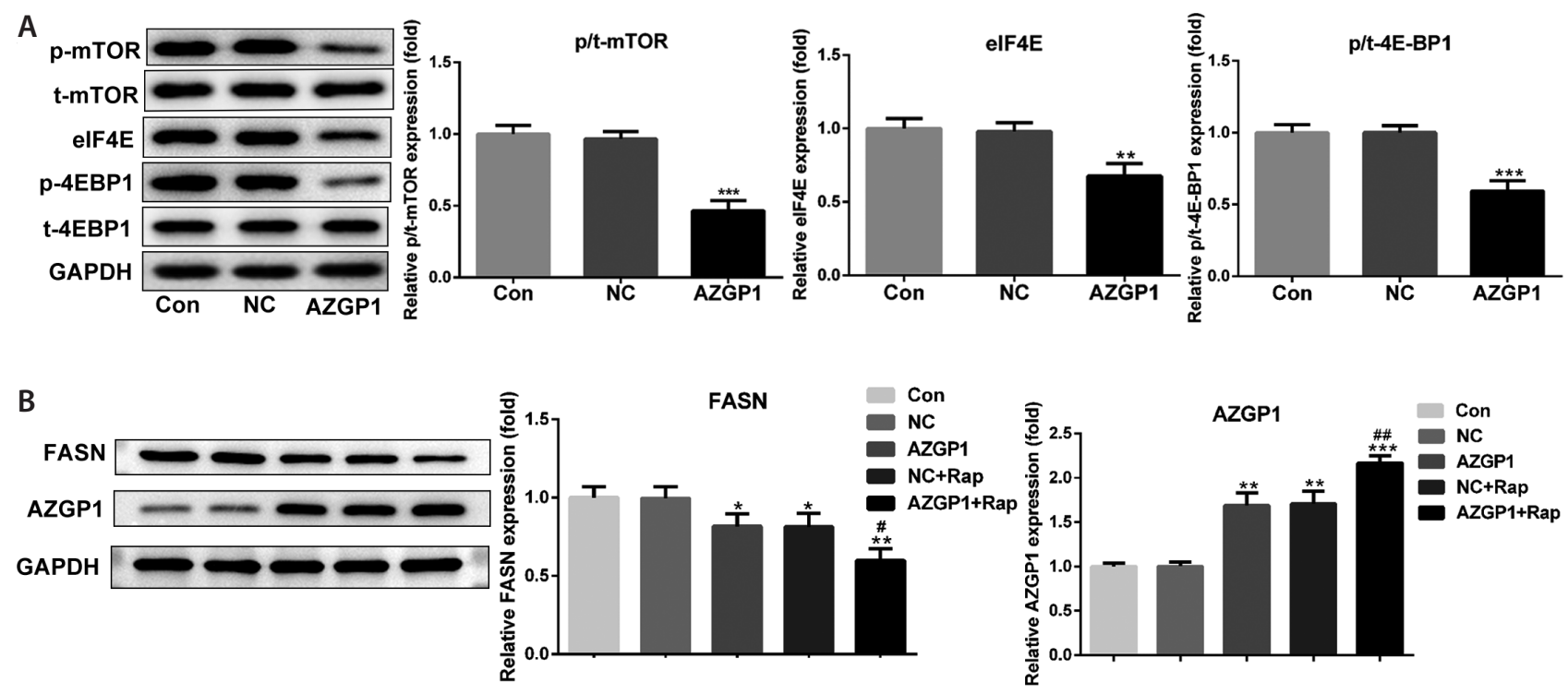

Figure 4. AZGP1 overexpression suppressed the expression of p/t-mTOR, eIF4E and p/t-4EBP1 in SW480 cells. A. The protein expressions of p-mTOR, t-mTOR, eIF4E and p-4EBP1 and t-4EBP1 in SW480 cells after treatment of overexpression-AZGP1 (AZGP1) and overexpression negative control (NC) were assessed using Western blotting assay. B. The protein expression of FASN and AZGP1 in SW480 cells in different groups after treated with AZGP1 overexpression or plus rapamycin (Rap). ${ }^{*} p<0.05,{ }^{* *} p<0.01$ and ${ }^{* * *} p<$ 0.001 vs. control (Con) or NC group; ${ }^{\#} p<0.05$ and ${ }^{\# \#} p<0.01$ vs AZGP1 or NC+Rap group.

Effects of overexpression-FASN on multiple malignant phenotypes of SW480 cells

Next, the results of Western blot and RT-qPCR indicated a marked increase both in protein and mRNA levels of FASN in the overexpression-FASN group comparing to control or
overexpression-NC group $(p<0.01$ and $p<0.001$; Fig. $5 \mathrm{~A}$ and B), while FASN expression was reduced significantly in the FASN shRNA group when compared with those in the control and shRNA-NC groups ( $p<0.01$; Fig. 5 C and D). The CCK-8 assay revealed that overexpression-FASN increased cell proliferation ( $p<0.05$ at 48 h, $p<0.01$ at 72 h; Fig. 6 A) but
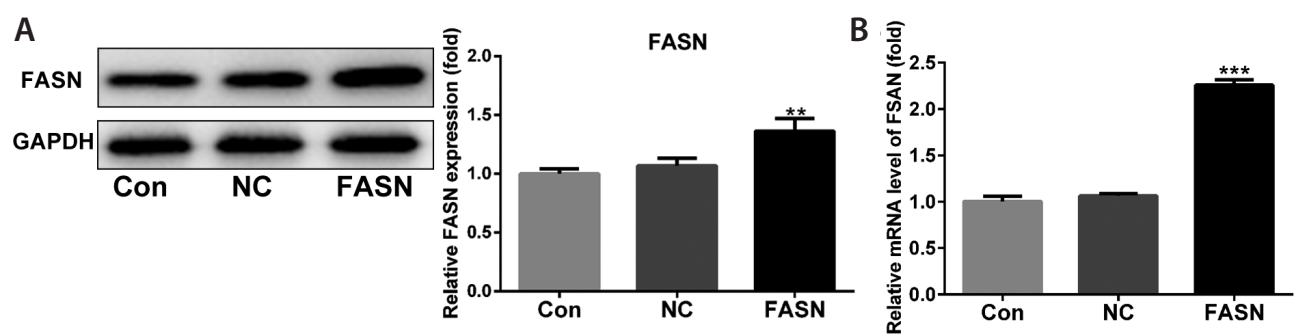

Figure 5. Detection of transfection efficiency of overexpression-FASN and FASN shRNA plasmids in SW480 cells. A, B. The protein and mRNA expression of FASN in SW480 cells
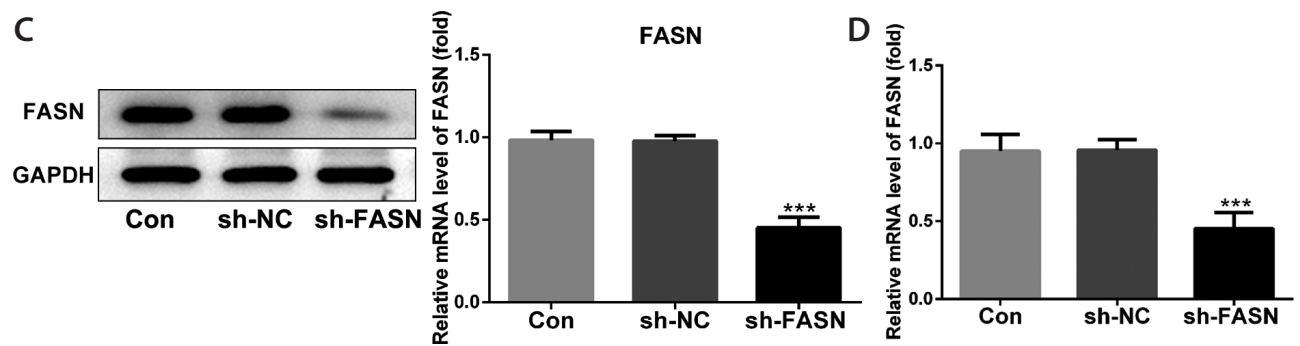
after transfection of overexpression-FASN (FASN) and overexpression negative control (NC), as dissected by Western blotting and qRT-PCR. ${ }^{* *} p<0.01$, $* * * p<0.001$ vs. control (Con) or NC groups. C, D. The protein and mRNA expression of FASN in SW480 cells after transfection with FASN shRNA (sh-FASN) and shRNA-NC (sh-NC) plasmids. ${ }^{* * *} p<0.001 v s$. control or sh-NC groups. 
overexpression-AZGP1 suppressed cell proliferation sharply when compared with the control or overexpression-NC group ( $p<0.01$ at $48 \mathrm{~h}, p<0.001$ at $72 \mathrm{~h}$; Fig. 6A). Interestingly, overexpression-FASN + overexpression-AZGP1 cotransfected SW480 cells exhibited a significantly increased growth rate compared with that of the overexpressionAZGP1-transfected cells ( $p<0.05$ at 72 h; Fig. 6A). Additionally, the growth rate in the overexpression-AZGP1 + FASN shRNA-co-transfected SW480 cells was dramatically inhibited when compared with the overexpression-AZGP1 group ( $p<0.05$ at $48 \mathrm{~h}, p<0.01$ at $72 \mathrm{~h}$; Fig. 6A). Therefore, overexpression of FASN markedly elevated and knockout of FASN effectively inhibited the proliferation/survival rate of SW 480 cells.

As shown in Fig. 6B, C and D, cell migration and invasion of SW480 cells were distinctly repressed with AZGP1 upregulation $(p<0.01)$ and promoted with FASN overexpression $(p<0.05)$, respectively. In comparison to overexpression-AZGP1 group, the migration and invasion abilities were enhanced after FASN was increased $(p<0.05)$ but suppressed after FASN was decreased $(p<0.05)$. As presented in Fig. 7, results of Western blotting disclosed that upregulation of AZGP1 and FASN obviously reduced and raised the protein levels of MMP-2 and MMP-9 $(p<$

A
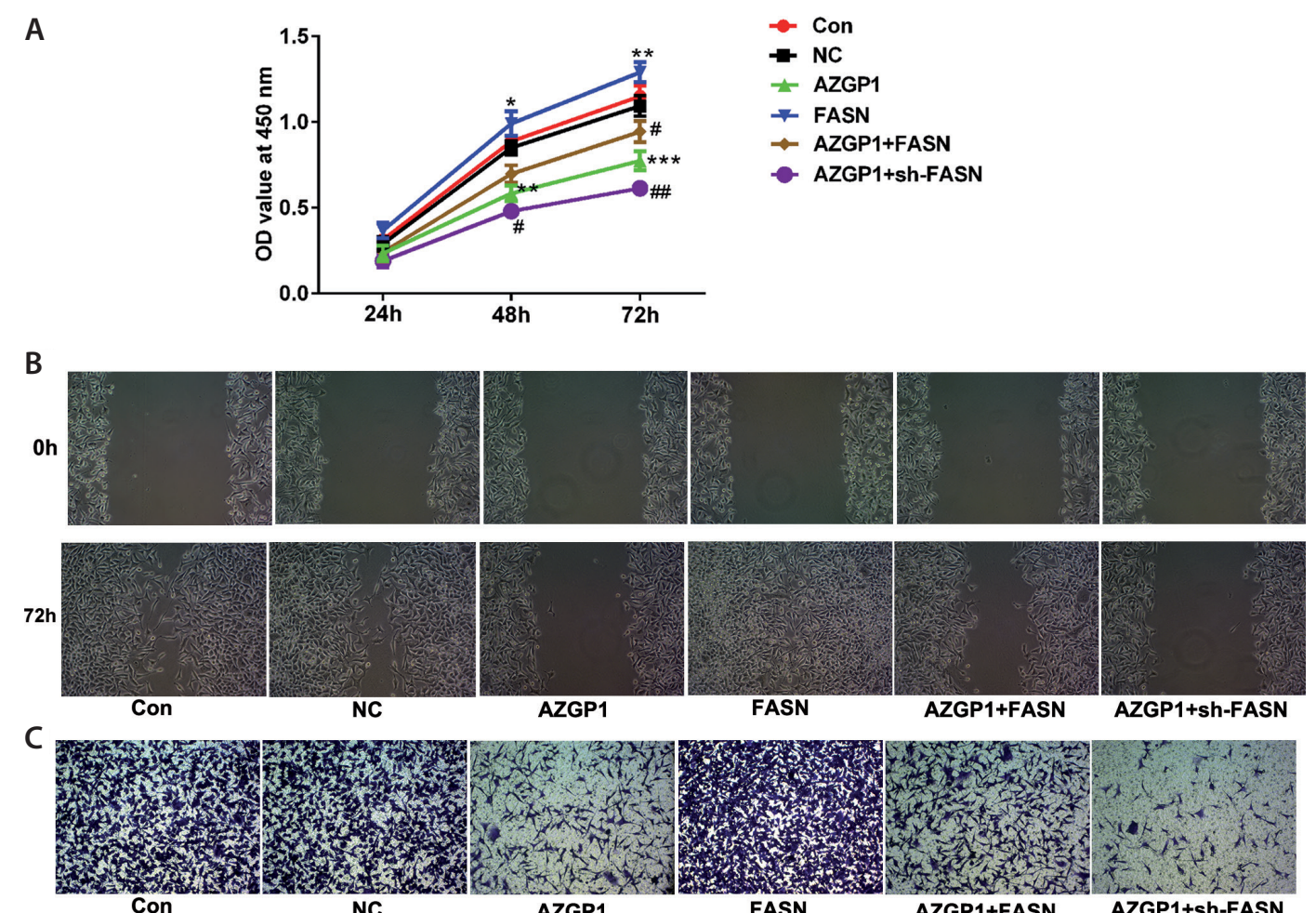

NC
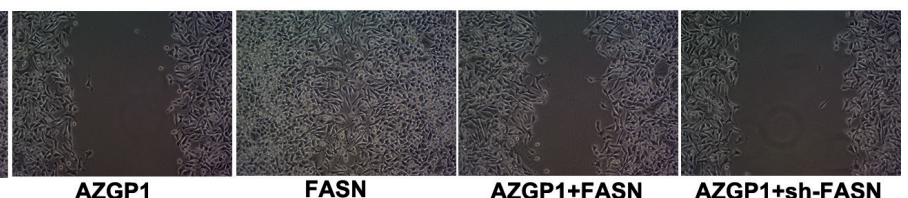

D

NC

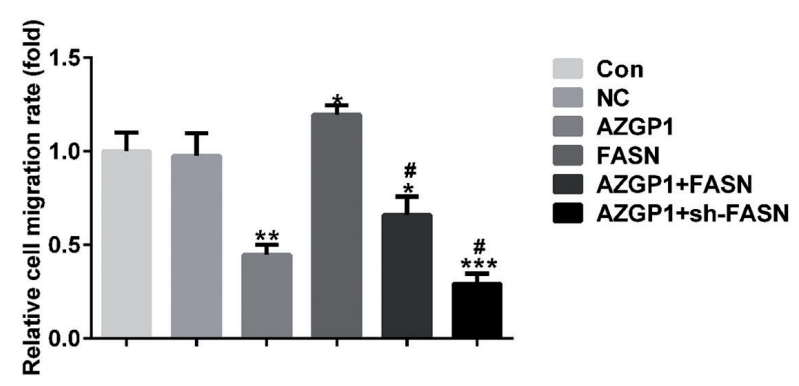

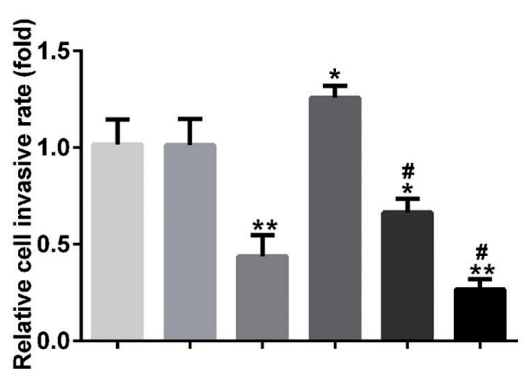

Figure 6. Overexpression of FASN elevated cell proliferation, invasion and migration which suppressed by AZGP1 overexpression. A. The effect of AZGP1 overexpression (AZGP1) and FASN overexpression (FASN) or FASN shRNA (sh-FASN) on cell proliferation using Cell Counting Kit-8 (CCK-8) assay. B, C. The influences of AZGP1 overexpression and FASN overexpression or FASN shRNA on cell migration and invasion by wound healing and Transwell assays. D. The statistic results from B and C. ${ }^{\star} p<0.05,{ }^{* *} p<0.01$ and ${ }^{* * *} p<0.001$ vs. control (Con) or overexpression negative control (NC) group; ${ }^{\#} p<0.05$ and ${ }^{\# \#} p<0.01$ vs. AZGP1 group. 


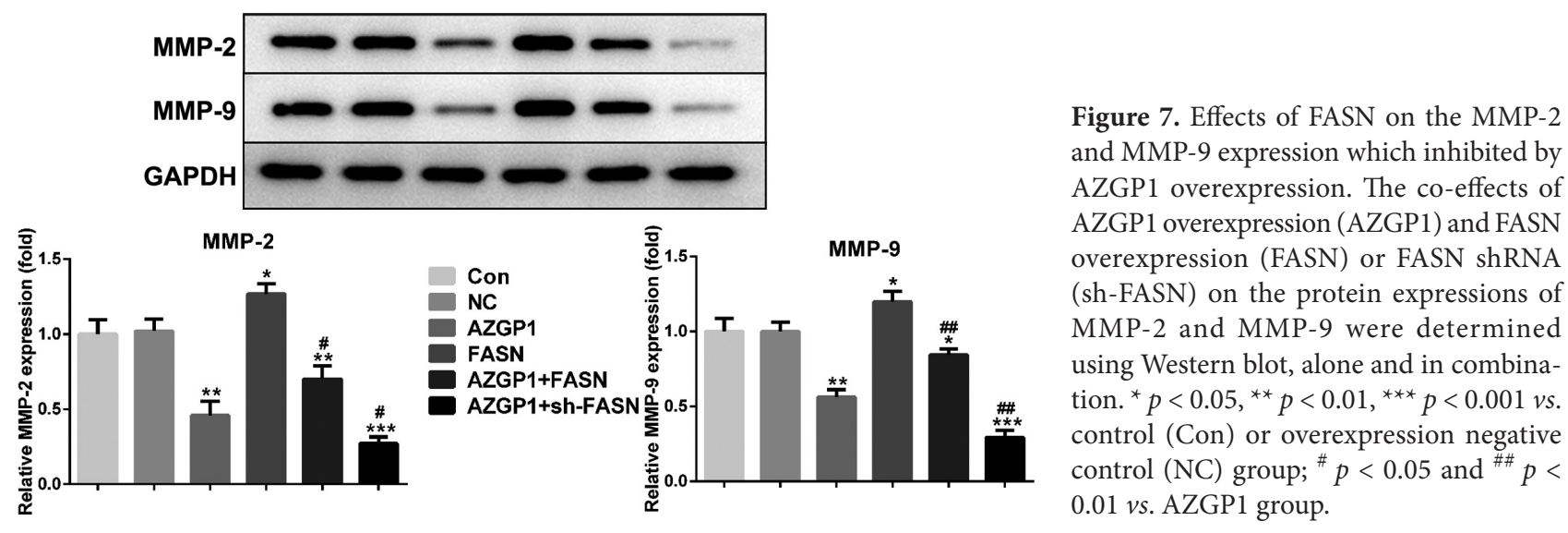

0.05 and $p<0.01)$. The expression of MMP-2 and MMP-9 were distinctly increased following FASN overexpression but remarkably downregulated following FASN silencing, when compared with overexpression-AZGP1 group $(p<$ 0.05 and $p<0.01$ ). These results displayed that FASN had a key role in the progression of CRC, and that the inhibition of FASN with shRNA-FASN would further decrease the malignant phenotypes of CRC under the background of overexpressing AZGP1.

\section{Discussion}

Malignant cells proliferate indefinitely and are prone to distant metastasis, which requires both sufficient energy and biosynthetic precursors to fuel cell division, invasion and migration (Yang et al. 2019). High expression of FASN may meet the needs of structural fatty acids and energy metabolism in tumor tissue survival, proliferation and invasion, which is similar to what we found in the current study that FASN is overexpressed in the CRC tissues with a decrease of AZGP1 expression, when compared with normal tissues (Fig. 1). FASN and AZGP1 are related with metabolic processes of several tumors and modulate cancer metabolism, which is currently an attractive anti-cancer approach. In the present study, the expression of FASN in CRC was affected by the AZGP1 and mTOR signaling pathway, and the interaction among three factors was important for regulating the malignant phenotype of CRC cells.

FASN is not expressed or micro-expressed in normal tissues (Li and Cheng 2014), while FASN expression is significantly up-regulated in various malignant tissues (Lettieri Barbato et al. 2014; Cui et al. 2017). In the obese CRC patients, the up-regulated expression of FASN is negatively correlated with the survival time of the patients (Ogino et al. 2008). A slight negative correlation between AZGP1 and FASN was found in the present study. Previous researches indicated that overexpression of AZGP1 displayed poor differentiation of tumors (Diez-Itza et al. 1993; Hale et al. 2001). Interestingly, after CRC cells were transfected with overexpression-AZGP1, the ability of proliferation, invasion and migration decreased obviously with down-regulation of MMP-2 and MMP-9 (Fig. 3). Past researches also reported that the mTOR signaling pathway could regulate cell cycle regulators (such as cyclins $\mathrm{D}, \mathrm{E}$, and $\mathrm{A}$ ), thereby accelerating the cell cycle and ultimately promoting tumor cell proliferation (Decker et al. 2003; Hong et al. 2008). 4EBP, a translation inhibitor, can bind to eIF4E, and block the normal function of recruiting the initiation complex. We found out that overexpression AZGP1 could suppress mTOR signaling via downregulating the downstream proteins $\mathrm{p}$-mTOR eIF4E and p-4EBP1 (Fig. 4). When mTOR signal activity is low, 4EBP is hypophosphorylated allowing for its binding to eIF4E, and thereby translation is blocked. While mTOR activity increases, it phosphorylates 4EBP, inhibiting its affinity for eIF4E and allowing cap-dependent translation to occur (Teleman et al. 2005). Thereafter, we further explored the interaction between the mTOR signaling pathway and FASN. Our findings showed that overexpression of AZGP1 reduced FASN expression significantly. Furthermore, the mTOR signal inhibitor, rapamycin, amplified this trend and re-downregulated FASN expression both in protein and mRNA levels.

In addition, the effects of overexpression AZGP1 on the malignant phenotype of CRC cells were attenuated by overexpressing FASN. In other words, FASN significantly elevated the proliferation, invasion and migration of CRC cells, which were suppressed by AZGP1 overexpression treatment. However, the inhibition of FASN further decreased the malignant phenotypes of CRC under the background of AZGP1 overexpression (Fig. 6). Meanwhile, a similar effect of FASN on the expressions of MMP2 and MMP9 could be observed in the CRC cells (Fig. 7). Therefore, AZGP1 suppresses CRC cell activities by regulating FASN via mTOR 
pathway, suggesting that AZGP1 and FASN may be the crucial metabolic controllers in CRC.

\section{Conclusion}

Due to that AZGP1 and FASN are linked to obesity, and obesity is linked to metabolic processes, so we speculate they may participate in tumorigenesis and development of CRC by regulating energy metabolism pathways. Further research is needed to verify the above supposition. Here, AZGP1 suppresses CRC cell activities by modulating FASN via mTOR pathway, hinting that AZGP1 and FASN may be the crucial metabolic controllers in CRC, and proper regulation of their activities may be a promising strategy for CRC treatment.

Funding. This study was supported by the Jurong people's livelihood science and technology project (No. SF2018807257).

Availability of data and materials. The datasets used and/or analyzed in the present study are available from the corresponding author upon reasonable request.

Conflict of interests. The authors declare that they have no competing interests.

\section{References}

Aleksandrova K, Schlesinger S, Fedirko V, Jenab M, Bueno-deMesquita B, Freisling H, Romieu I, Pischon T, Kaaks R, Gunter MJ, et al. (2017): Metabolic mediators of the association between adult weight gain and colorectal cancer: data from the european prospective investigation into cancer and nutrition (EPIC) cohort. Am. J. Epidemiol. 185, 751-764 https://doi.org/10.1093/aje/kww194

Cai Y, Wang J, Zhang L, Wu D, Yu D, Tian X, Liu J, Jiang X, Shen Y, Zhang L, Ren M, Huang P (2015): Expressions of fatty acid synthase and HER2 are correlated with poor prognosis of ovarian cancer. Med. Oncol. 32, 391 https://doi.org/10.1007/s12032-014-0391-Z

Chang L, Tian X, Lu Y, Jia M, Wu P, Huang P (2014): Alpha2-glycoprotein 1(AZGP1) regulates biological behaviors of LoVo cells by down-regulating mTOR signaling pathway and endogenous fatty acid synthesis. PLoS One 9, e99254 https://doi.org/10.1371/journal.pone.0099254

Chang L, Fang S, Chen Y, Yang Z, Yuan Y, Zhang J, Ye L, Gu W (2019): Inhibition of FASN suppresses the malignant biological behavior of non-small cell lung cancer cells via deregulating glucose metabolism and AKT/ERK pathway. Lipids Health Dis. 18, 118 https://doi.org/10.1186/s12944-019-1058-8

Cui Y, Xing P, Wang Y, Liu M, Qiu L, Ying G, Li B (2017): NADPH accumulation is responsible for apoptosis in breast cancer cells induced by fatty acid synthase inhibition. Oncotarget $\mathbf{8}$, 32576-32585 https://doi.org/10.18632/oncotarget.15936
Decker T, Hipp S, Ringshausen I, Bogner C, Oelsner M, Schneller F, Peschel C (2003): Rapamycin-induced G1 arrest in cycling $\mathrm{B}-\mathrm{CLL}$ cells is associated with reduced expression of cyclin D3, cyclin E, cyclin A, and survivin. Blood 101, 278-285 https://doi.org/10.1182/blood-2002-01-0189

Diez-Itza I, Sanchez LM, Allende MT, Vizoso F, Ruibal A, LopezOtin C (1993): Zn-alpha 2-glycoprotein levels in breast cancer cytosols and correlation with clinical, histological and biochemical parameters. Eur. J. Cancer 29A, 1256-1260 https://doi.org/10.1016/0959-8049(93)90068-Q

Fang G, Zhang P, Liu J, Zhang X, Zhu X, Li R, Wang H (2019): Inhibition of GSK-3beta activity suppresses HCC malignant phenotype by inhibiting glycolysis via activating AMPK/mTOR signaling. Cancer Lett. 46, 11-26 https://doi.org/10.1016/j.canlet.2019.08.003

Gao N, Flynn DC, Zhang Z, Zhong XS, Walker V, Liu KJ, Shi X, Jiang BH (2004): G1 cell cycle progression and the expression of G1 cyclins are regulated by PI3K/AKT/mTOR/p70S6K1 signaling in human ovarian cancer cells. Am. J. Physiol. Cell Physiol. 287, C281-291 https://doi.org/10.1152/ajpcell.00422.2003

Hale LP, Price DT, Sanchez LM, Demark-Wahnefried W, Madden JF (2001): Zinc alpha-2-glycoprotein is expressed by malignant prostatic epithelium and may serve as a potential serum marker for prostate cancer. Clin. Canc. Res. 7, 846-853

Hong F, Larrea MD, Doughty C, Kwiatkowski DJ, Squillace R, Slingerland JM (2008): mTOR-raptor binds and activates SGK1 to regulate p27 phosphorylation. Mol. Cell 30, 701-711 https://doi.org/10.1016/j.molcel.2008.04.027

Jemal A, Siegel R, Ward E, Hao Y, Xu J, Murray T, Thun MJ (2008): Cancer statistics, 2008. CA Cancer J. Clin. 58, 71-96 https://doi.org/10.3322/CA.2007.0010

Larsson SC, Wolk A (2007): Obesity and colon and rectal cancer risk: a meta-analysis of prospective studies. Am. J. Clin. Nutr. 86, $556-565$ https://doi.org/10.1093/ajcn/86.3.556

Lettieri Barbato D, Vegliante R, Desideri E, Ciriolo MR (2014): Managing lipid metabolism in proliferating cells: new perspective for metformin usage in cancer therapy. Biochim. Biophys. Acta 1845, 317-324

https://doi.org/10.1016/j.bbcan.2014.02.003

Li J, Cheng JX (2014): Direct visualization of de novo lipogenesis in single living cells. Sci. Rep. 4, 6807

https://doi.org/10.1038/srep06807

Li N, Bu X, Tian X, Wu P, Yang L, Huang P (2012): Fatty acid synthase regulates proliferation and migration of colorectal cancer cells via HER2-PI3K/Akt signaling pathway. Nutr. Cancer 64, 864-870 https://doi.org/10.1080/01635581.2012.701704

Marin de Mas I, Torrents L, Bedia C, Nielsen LK, Cascante M, Tauler R (2019): Stoichiometric gene-to-reaction associations enhance model-driven analysis performance: Metabolic response to chronic exposure to Aldrin in prostate cancer. BMC Genomics 20, 652 https://doi.org/10.1186/s12864-019-5979-4

Marrades MP, Martinez JA, Moreno-Aliaga MJ (2008): ZAG, a lipid mobilizing adipokine, is downregulated in human obesity. J. Physiol. Biochem. 64, 61-66 
https://doi.org/10.1007/BF03168235

Nakazato K, Mogushi K, Kayamori K, Tsuchiya M, Takahashi KI, Sumino J, Michi Y, Yoda T, Uzawa T (2019): Glucose metabolism changes during the development and progression of oral tongue squamous cell carcinomas. Oncol. Lett. 18, 1372-1380 https://doi.org/10.3892/ol.2019.10420

Nozawa H, Watanabe T, Nagawa H (2007): Phosphorylation of ribosomal p70 S6 kinase and rapamycin sensitivity in human colorectal cancer. Cancer Lett. 251, 105-113 https://doi.org/10.1016/j.canlet.2006.11.008

Ogino S, Kawasaki T, Ogawa A, Kirkner GJ, Loda M, Fuchs CS (2007): Fatty acid synthase overexpression in colorectal cancer is associated with microsatellite instability, independent of $\mathrm{CpG}$ island methylator phenotype. Human Pathol. 38, 842-849 https://doi.org/10.1016/j.humpath.2006.11.018

Ogino S, Nosho K, Meyerhardt JA, Kirkner GJ, Chan AT, Kawasaki T, Giovannucci EL, Loda M, Fuchs CS (2008): Cohort study of fatty acid synthase expression and patient survival in colon cancer. J. Clin. Oncol. 26, 5713-5720 https://doi.org/10.1200/JCO.2008.18.2675

Russell ST, Zimmerman TP, Domin BA, Tisdale MJ (2004): Induction of lipolysis in vitro and loss of body fat in vivo by zinc-alpha2-glycoprotein. Biochim. Biophys. Acta 1636, 59-68 https://doi.org/10.1016/j.bbalip.2003.12.004

Tada T, Ohkubo I, Niwa M, Sasaki M, Tateyama H, Eimoto T (1991): Immunohistochemical localization of Zn-alpha 2-glycoprotein in normal human tissues. J. Histochem. Cytochem. 39, $1221-1226$ https://doi.org/10.1177/39.9.1918940

Teleman AA, Chen YW, Cohen SM (2005): 4E-BP functions as a metabolic brake used under stress conditions but not during normal growth. Genes Develop. 19, 1844-1848 https://doi.org/10.1101/gad.341505

Wang Y, Kuhajda FP, Li JN, Pizer ES, Han WF, Sokoll LJ, Chan DW (2001): Fatty acid synthase (FAS) expression in human breast cancer cell culture supernatants and in breast cancer patients. Cancer Lett. 167, 99-104 https://doi.org/10.1016/S0304-3835(01)00464-5

Yang J, Ren B, Yang G, Wang H, Chen G, You L, Zhang T, Zhao Y (2019): The enhancement of glycolysis regulates pancreatic cancer metastasis. Cell. Mol. Life Sci. 77, 305-321 https://doi.org/10.1007/s00018-019-03278-Z

Zaytseva YY, Rychahou PG, Gulhati P, Elliott VA, Mustain WC, O'Connor K, Morris AJ, Sunkara M, Weiss HL, Lee EY, Evers BM (2012): Inhibition of fatty acid synthase attenuates CD44associated signaling and reduces metastasis in colorectal cancer. Cancer Res. 72, 1504-1517 https://doi.org/10.1158/0008-5472.CAN-11-4057

Zhang L, Yang Y, Chai L, Bu H, Yang Y, Huang H, Ran J, Zhu Y, Li L, Chen F, Li W (2019): FRK plays an oncogenic role in nonsmall cell lung cancer by enhancing the stemness phenotype via induction of metabolic reprogramming. Int. J. Cancer. 146, 208-222

https://doi.org/10.1002/ijc.32530

Received: November 4, 2019

Final version accepted: December 14, 2019 